\title{
Efficacy of hybrid 2-port hand-assisted laparoscopic surgery (Mukai's operation) for patients with primary colorectal cancer
}

\author{
MASAYA MUKAI $^{1}$, KYOKO KISHIMA ${ }^{1}$, TAKAYUKI TAJIMA ${ }^{1}$, TATSUHIKO HOSHIKAWA ${ }^{1}$, \\ NAOKI YAZAWA ${ }^{1}$, HIROSHI FUKUMITSU ${ }^{1}$, KAZUTAKE OKADA ${ }^{1}$, \\ KYOUJI OGOSHI $^{2}$ and HIROYASU MAKUUCHI ${ }^{2}$
}

\author{
${ }^{1}$ Department of Surgery, Tokai University Hachioji Hospital, Ishikawa-cho 1838, Hachioji, Tokyo, 192-0032; \\ ${ }^{2}$ Department of Surgery, Tokai University School of Medicine, Bohseidai, Isehara, Kanagawa 259-1193, Japan
}

Received June 15, 2009; Accepted July 24, 2009

DOI: 10.3892/or_00000514

\begin{abstract}
In this study, a total of 108 patients with primary colorectal cancer who underwent hybrid 2-port hand-assisted laparoscopic surgery (HALS) were classified as 58 patients with colon cancer and 50 patients with rectal cancer. The mean operating time, mean blood loss, postoperative complications, and mean postoperative hospital stay were compared between the two groups. In patients who underwent colon cancer surgery, the mean operating time was $2 \mathrm{~h}$ and $26 \mathrm{~min}$, the mean blood loss was $166.3 \mathrm{ml}$, and the postoperative complications were wound infection in $5 / 58$ patients $(8.6 \%)$, postoperative ileus in 3 patients $(5.2 \%)$, and anastomotic stricture in 1 patient $(1.7 \%)$. There was no anastomotic leakage and no conversion to conventional open laparotomy. The mean postoperative hospital stay was 12.6 days. In patients who underwent rectal cancer surgery, the mean operating time was $3 \mathrm{~h}$ and $38 \mathrm{~min}$, the mean blood loss was $238.8 \mathrm{ml}$, and the postoperative complications consisted of wound infection in $6 / 50$ patients $(12.0 \%$ ), anastomotic leakage in $3 / 35$ patients $(8.6 \%)$, anastomotic stricture in $3 / 47$ patients $(6.4 \%)$, postoperative ileus in $3 / 50$ patients $(6.0 \%)$, and conversion to conventional open laparotomy in $1 / 50$ patients $(2.0 \%)$. A covering stoma was constructed during surgery in $12 / 47$ patients $(25.5 \%)$. The mean postoperative hospital stay was
\end{abstract}

Correspondence to: Dr Masaya Mukai, Department of Surgery, Tokai University Hachioji Hospital, Ishikawa-cho 1838, Hachioji, Tokyo 192-0032, Japan

E-mail: mukai.masaya@hachioji-hosp.tokai.ac.jp

Abbreviations: LACS, laparoscopy-assisted colorectal surgery; HALS, hand-assisted laparoscopic surgery; hybrid 2-port HALS, hybrid 2-port hand-assisted laparoscopic surgery; FEEA, functioning end-to-end anastomosis; DST, double-stapling technique; LAR, low anterior resection

Key words: colorectal cancer, laparoscopy-assisted colorectal surgery, hand-assisted colorectal surgery, hybrid hand-assisted laparoscopic surgery
19.1 days. These results suggest that hybrid 2-port HALS (Mukai's operation) could become a standard method for the treatment of colorectal cancer, and that the long-term outcome should be compared in detail with that of standard laparotomy in the future.

\section{Introduction}

Less invasive surgery such as laparoscopy-assisted colorectal surgery (LACS) has become popular in recent years, and its indications have expanded markedly from additional resection in patients with stage I colorectal cancer to radical resection in patients with stage II/III cancer and palliative surgery for patients with advanced stage IV disease (1-5). Unlike Western countries, where hand-assisted laparoscopic surgery (HALS) and hybrid HALS combined with open manipulation are performed (6-9), the main type of surgery employed in Japan is pure LACS with 5-6 ports including a camera port for manipulation and a small incision of 35-45 $\mathrm{mm}$ (10-12). However, at least 3 surgeons experienced with smooth camera operation are required for pure LACS since the operation is mostly performed by the operator and first assistant manipulating four forceps. Pure LACS has the following disadvantages compared with ordinary open laparotomy: i) poor palpation/tactile sensation, ii) limited applicability with respect to large and heavy tumors, ii) difficulty in assessing the total operating field, iv) a longer operating time as laparoscopic manipulation while observing the monitor is the main procedure, and v) a requirement to acquire specific skills and pass the certification exam in Japan. In addition, LACS cannot become the standard operation even at a relatively large general hospital since it requires several experienced surgeons to shorten the duration of the procedure and due to the lack of anesthesiologists. In fact, some institutions have withdrawn from the routine use of LACS. According to the results of a national questionnaire survey of members of the Japan Society for Endoscopic Surgery in 2008, LACS for colorectal diseases was performed on a total of nearly 13,500 patients whereas routine laparotomy was performed for nearly 70,300 patients, suggesting that LACS procedures in Japan account for approximately one fifth of the patients managed by routine laparotomy $(13,14)$. The use of LACS should be more 
prevalent in Japan, but its adoption rate has slowed since the institutional stance seems to have become polarized concerning the use of LACS. Introduction of LACS might be considered at institutions where routine laparotomy is the standard method, but the internal standardization/routinization by the operator and the first assistant experienced with pure LACS is an important issue to be solved. We therefore devised hybrid 2-port HALS (Mukai's operation) that employs a hand access site measuring $45-55 \mathrm{~mm}$ to solve these issues, and we previously reported various procedures for patients with colorectal cancer $(5,15-17)$. The advantages of our methods include i) safe palpation and good tactile sensation, ii) full grasping manipulation by the left hand that enables protective and smooth handling of even a large and heavy tumor, iii) manipulation can be carried out by at least 2 surgeons, iv) a shorter operating time is required since it is an extension of standard laparotomy, and v) less time is required to become experienced with the procedure. Unlike pure LACS, open surgery can be used, not only for anastomosis, but also for complete closure of the mesocolon/mesorectum after colorectal resection, ligation/dissection of large vessels. In addition, lymph node excision can be achieved by routine methods, and the hybrid method is safely applicable for patients with ileus or those who need resection of tumors infiltrating other organs $(5,16,17)$. When the left hand is inserted distal to the tumor, the procedure is not inferior to routine laparotomy even with regard to dissection in layers. In addition, manipulation at the pelvic floor/posterior surface of the prostate has to be performed almost blind during routine laparotomy due to difficulty in viewing these sites, but it can be safely performed while viewing magnified images on a monitor when hybrid 2-port HALS is employed. Therefore, it may be indicated for almost all patients.

However, the results of surgery and reports of complications have not yet been published for hybrid 2-port HALS (Mukai's operation) in patients with colorectal cancer. Accordingly, the aim of this study was to review the surgical results of hybrid 2-port HALS and the short-term outcome such as postoperative complications and hospital stay in patients with colorectal cancer.

\section{Patients and methods}

The subjects enrolled in this study were a total of 108 patients with primary colorectal cancer (stage 0/I, 36 patients; stage II, 38 patients; stage III, 30 patients; stage IV, 4 patients) who underwent hybrid 2-port HALS from June 2007 to June 2009. They were classified as 58 patients with colon cancer and 50 patients with rectal cancer (Table I). This type of surgery was indicated in the following patients: i) no history of major laparotomy, ii) ability to tolerate general anesthesia for $\sim 2 \mathrm{~h}$, iii) no major concomitant diseases such as heart and/or lung disease, iv) preferably a P.S. of 0-1 and age $<75$ years, and v) no preoperatively detectable metastasis to the lateral lymph nodes and no invasion of the pelvic cavity in patients with rectal cancer $(15,16)$.

Colon cancer patients. One of the following 4 procedures was performed in a total of 58 patients with colon cancer (cecum, 13 patients; ascending colon, 15 patients; transverse
Table I. Hybrid 2-port HALS (Mukai's operation) in 108 patients with primary colorectal cancer.

Total 108 patients

Stage: 0/1, 36 cases; II, 38 cases; III, 30 cases; IV , 4 cases

Colon (58 patients)

Location: C, 13 cases; A, 15 cases; T, 10 cases, D, 5 cases,

S, 15 cases

$\begin{array}{lr}\text { Right hemicolectomy } & 30 \text { cases } \\ \text { Transverse colectomy } & 8 \text { cases } \\ \text { Left hemicolectomy } & 5 \text { cases } \\ \text { Sigmoidectomy } & 15 \text { cases }\end{array}$

Rectum (50 patients)

Location: Rs, 16 cases; Ra, 20 cases; Rb, 14 cases

Anterior resection

16 cases

Low anterior resection

31 cases

Miles' operation

3 cases

C, cecum; A, ascending colon; T, transverse colon; D, descending colon; S, sigmoid colon. Rs, rectosigmoid; Ra, rectum/above the peritoneal reflection; $\mathrm{Rb}$, rectum/below the peritoneal reflection.

colon, 10 patients; descending colon, 5 patients; and sigmoid colon, 15 patients) (Table I).

a) Right hemicolectomy was carried out in 30 patients. First, a small longitudinal upper median mini-incision of 45-55 mm was made in the upper abdomen (Fig. 1A). After attaching the hand access device (Lapdisc ${ }^{\circledR}$, regular type, 120x120 mm; Hakkou Co., Ltd., Nagano, Japan) to the incision site, a pneumoperitoneum was created with the patient in the head-down and lithotomy/right superior oblique position. The right ureter was confirmed after sufficient mobilization of the cecum, and the transverse colon was separated from the curvature of the liver to the anterior surface of the duodenum. The middle colic artery (A) and vein (V) were ligated/dissected by open surgery to perform D2 or D3 resection. The mesocolon was closed prior to creation of a functioning end-to-end anastomosis (FEEA) extracorporeally (Table I) (17).

b) Transverse colectomy was carried out in 8 patients. Either a small longitudinal upper median mini-incision of $\sim 45-55 \mathrm{~mm}$ (Fig. 1A) or a small longitudinal upper leftpararectal mini-incision of $\sim 45-55 \mathrm{~mm}$ (Fig. 1B) was made in the upper abdomen. After attaching the access device, a pneumoperitoneum was created with the patient in the headup and lithotomy position. The bases of the left and right sides of the colon were dissected/mobilized after a pneumoperitoneum was established, and the middle colic A/V were ligated/dissected as an open procedure to perform D2,3 resection. The mesocolon was closed prior to performing extracorporeal FEEA (Table I) (17).

c) Left hemicolectomy was performed in 5 patients. A small longitudinal upper left-pararectal mini-incision of 45-55 mm was made in the upper abdomen (Fig. 1B). Sufficient dissection/mobilization of the bases of the left and splenic flexure sides of the colon were carried out after a 


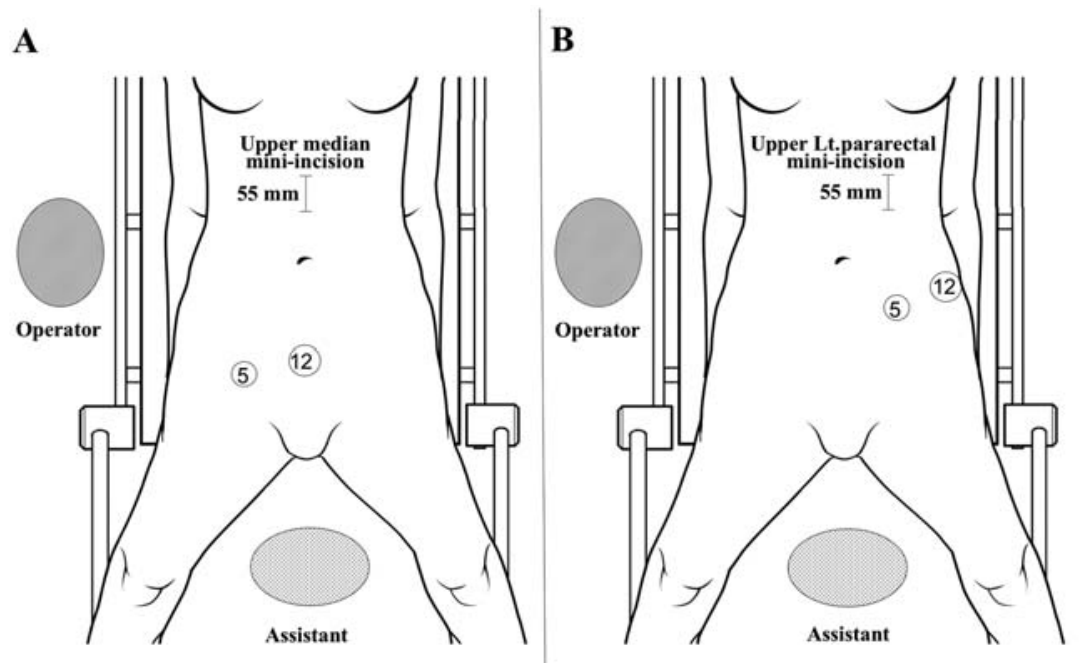

Figure 1. Illustrations of patients in the lithotomy position and an overall view of the abdomen, including the mini-incision and the positions of the 2 ports for right hemicolectomy $(\mathrm{A})$, transverse colectomy (A and $\mathrm{B})$, and left hemicolectomy $(\mathrm{B})$.
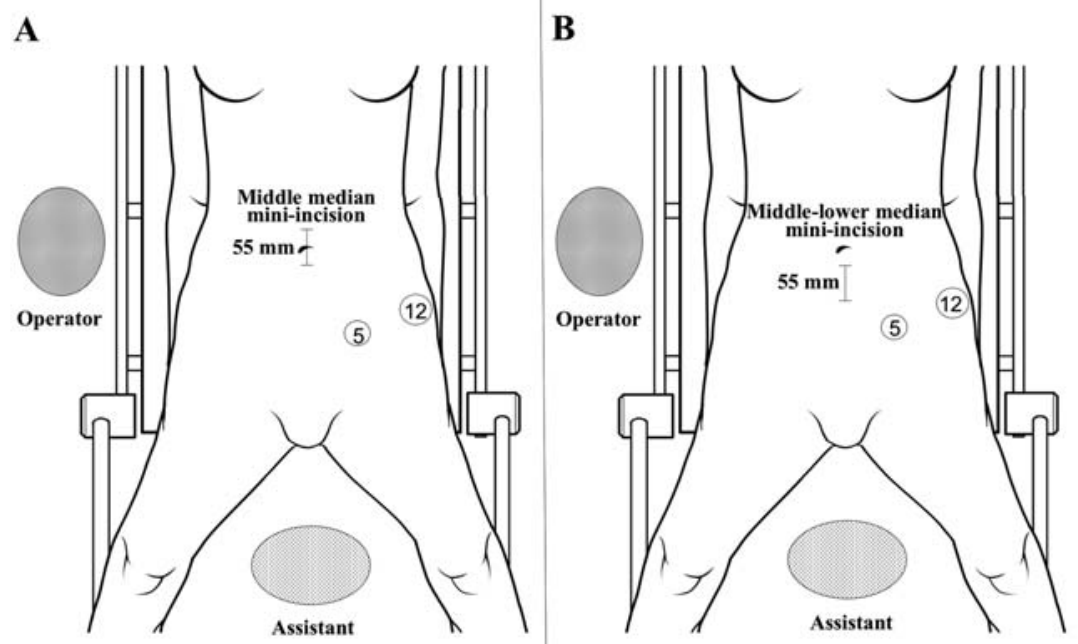

Figure 2. Illustrations of patients in the lithotomy position and an overall view of the abdomen, including the mini-incision and the 2 ports for sigmoidectomy (A and $\mathrm{B}$ ).

pneumoperitoneum was established with the patient in the head-up and lithotomy/left superior oblique position. Then the left colic $\mathrm{A} / \mathrm{V}$ were ligated/dissected as an open procedure to perform D2,3 resection. The mesocolon was closed prior to extracorporeal FEEA (Table I) (5).

d) Sigmoidectomy was performed in 15 patients. Either a small longitudinal mini-incision of $\sim 45-55 \mathrm{~mm}$ was created in the midline at the para-umbilical region (Fig. 2A) or a small longitudinal mini-incision of $\sim 45-55 \mathrm{~mm}$ was made in the midline of the lower abdomen (Fig. 2B). Then the region from the sigmoid mesocolon to the mesorectum was sufficiently dissected/mobilized after a pneumoperitoneum was created with the patient in the head-down and lithotomy/ left anterior oblique position to allow confirmation of the left ureter. Either the inferior mesenteric artery/basal vein or distal left colic A/V were ligated/dissected to perform D2,3 resection. After closing the mesocolon and mesorectum, reconstruction was performed by either extracorporeal FEEA or by rectal transection with a 51-mm curved cutter (Greencartridge; Ethicon Endo-Surgery, Puerto Rico, USA), followed by intracorporeal anastomosis with a Proxymate ILS Endopath, Endo Circular Stapler (ECS33; Ethicon EndoSurgery) double-stapling technique (DST) (Table I) (16).

Rectal cancer patients. One of the following 2 procedures was performed in a total of 50 patients with rectal cancer [rectosigmoid (Rs) in 16 patients, rectum/above the peritoneal reflection $(\mathrm{Ra})$ in 20 patients, and rectum/below the peritoneal reflection $(\mathrm{Rb})$ in 14 patients].

e) A total of 31 patients had low anterior resection (LAR) and 16 patients underwent anterior resection. Either a small transverse mini-incision of $\sim 45-55 \mathrm{~mm}$ was made above the superior border of the pubic bone (Fig. 3A) or a longitudinal mini-incision of $\sim 45-55 \mathrm{~mm}$ was made in the lower median 
A

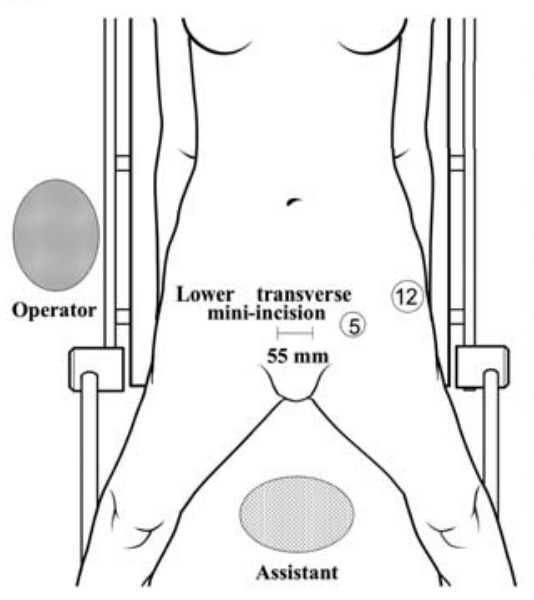

B

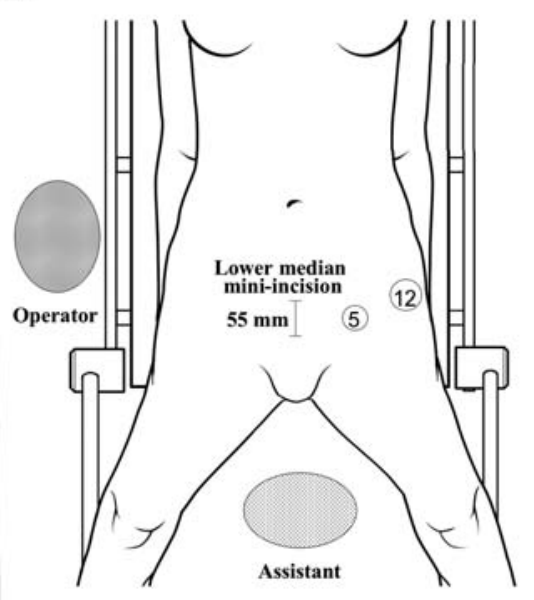

Figure 3. Illustrations of patients in the lithotomy position and an overall view of the abdomen, including the mini-incision and the 2 ports for low anterior resection or Miles' operation in patients with rectal cancer (A and $\mathrm{B})$.

Table II. Results and complications of hybrid 2-port HALS (Mukai's operation) in 58 patients with colon cancer.

\begin{tabular}{ll}
\hline Mean operating time & $2 \mathrm{~h} 26 \mathrm{~min}$ (minimum $1 \mathrm{~h} 10$ min, maximum $4 \mathrm{~h} 8 \mathrm{~min})$ \\
Mean blood loss & $166.3 \mathrm{ml}$ (minimum $8 \mathrm{ml}$, maximum $1100 \mathrm{ml})$ \\
Mean postoperative hospital stay & 12.6 days (minimum 8 days, maximum 46 days) \\
Complications & $5 / 58$ cases $(8.6 \%$, initially $3 / 20$ cases) \\
Wound infection & $3 / 58$ cases $(5.2 \%)$ \\
Postoperative ileus & $1 / 58$ cases $(1.7 \%)$ \\
Anastomotic stricture & $0 / 58$ cases $(0.0 \%)$ \\
Leakage & $0 / 58$ cases $(0.0 \%)$ \\
Conversion to conventional surgery &
\end{tabular}

region (Fig. 3B). Then the region from the sigmoid colon/ sigmoid mesocolon to the mesorectum was adequately dissected out/mobilized after a pneumoperitoneum was created with the patient in the head-down and lithotomy/left anterior oblique position. The left ureter was confirmed, and the entire rectum from the anterior surface of the sacrum to the posterior surface of the bladder was mobilized along with a total mesorectal excision to reach the pelvic floor. The distal left colic A/V were also ligated/dissected to perform D2 resection. After transecting the rectum with a 51-mm curved cutter (Green-cartridge) or an Endopath stapler (Ethlon 60-mm Green/Gold cartridge; Ethicon Endo-Surgery), intracorporeal anastomosis was performed with an ECS33 and DST to close the mesocolon/mesorectum and reconstruct the pelvic floor (Table I) (15).

f) Abdomino-perineal resection (Miles' operation) was performed in 3 patients. Either a small transverse mini-incision of $\sim 45-55 \mathrm{~mm}$ was made to the superior border of the pubic bone (Fig. 3A) or a longitudinal mini-incision of $\sim 45-55 \mathrm{~mm}$ was made in the lower median region (Fig. 3B). Then the region from the sigmoid colon/sigmoid mesocolon to the mesorectum was sufficiently dissected/mobilized after a pneumoperitoneum was created with the patient in the headdown and lithotomy/left anterior oblique position. After confirming both ureters, the entire rectum from the anterior surface of the sacrum to the posterior surface of the bladder was mobilized along with total mesorectal excision to reach the pelvic floor muscles in the perineal region. The distal left colic A/V were ligated/dissected to perform D2 resection. The perineal wound was closed after rectal amputation to reconstruct the pelvic floor, and a permanent sigmoid endocolostomy was constructed (Table I) (15).

Two-port HALS with a 5-mm surgical port and a $12-\mathrm{mm}$ camera port was employed for endoscopic procedures with all of the above operations (Mukai's operation) (Figs. 1-3). The patients were classified into two groups (colon: a-d and rectum: e-f), which were compared with regard to the mean operating time, mean blood loss, postoperative complications, and mean postoperative hospital stay.

\section{Results}

For colon cancer surgery, the mean operating time was $2 \mathrm{~h}$ and $26 \mathrm{~min}$ (ranging from $1 \mathrm{~h}$ and $10 \mathrm{~min}$ to $4 \mathrm{~h}$ and $8 \mathrm{~min}$ ), and the mean blood loss was $166.3 \mathrm{ml}$ (range 8-1100 ml) (Table II). Postoperative complications were wound infection in $5 / 58$ patients $(8.6 \%$, initially $3 / 20$ patients), postoperative ileus in 3 patients $(5.2 \%)$, and anastomotic stricture in one 
Table III. Results and complications of hybrid 2-port HALS (Mukai's operation) in 50 patients with rectal cancer.

\begin{tabular}{ll}
\hline Mean operating time & $3 \mathrm{~h} 38 \mathrm{~min}$ (minimum $1 \mathrm{~h} 57 \mathrm{~min}$, maximum $7 \mathrm{~h} 7 \mathrm{~min})$ \\
Mean blood loss & $238.8 \mathrm{ml}$ (minimum $15 \mathrm{ml}$, maximum $2400 \mathrm{ml})$ \\
$\begin{array}{l}\text { Mean postoperative hospital stay } \\
\text { Complications }\end{array}$ & 19.1 days (minimum 9 days, maximum 167 days) \\
Wound infection & $6 / 50$ cases $(12.0 \%$, initially $4 / 20$ cases) \\
Leakage & $3 / 35$ cases $(8.6 \%$, excluding Miles' surgery 3 cases + intraoperative covering \\
& stoma 12 cases $)$ \\
Anastomotic stricture & $3 / 47$ cases $(6.4 \%$, excluding Miles' surgery 3 cases) \\
Postoperative ileus & $3 / 50$ cases $(6.0 \%)$ \\
Conversion to conventional surgery & $1 / 50$ cases $(2.0 \%)$ \\
Intraoperative covering stoma & $12 / 47$ cases $(25.5 \%, \mathrm{Rs}, 0 / 16 ; \mathrm{Ra}, 3 / 20 ; \mathrm{Rb}, 9 / 11)$ \\
\hline
\end{tabular}

Rs, rectosigmoid; Ra, rectum/above the peritoneal reflection; Rb, rectum/below the peritoneal reflection.

patient $(1.7 \%)$. There was no anastomotic leakage and no conversion to conventional open laparotomy. The mean postoperative hospital stay was 12.6 days, range 8-46 days (Table II).

For rectal cancer surgery, the mean operating time was $3 \mathrm{~h}$ and $38 \mathrm{~min}$ (range $1 \mathrm{~h}$ and $57 \mathrm{~min}-7 \mathrm{~h}$ and $7 \mathrm{~min}$ ) and the mean blood loss was $238.8 \mathrm{ml}$ (ranging from 15 to 2,400 ml) (Table III). Postoperative complications were wound infection in $6 / 50$ patients $(12.0 \%$, initially $4 / 20$ patients), anastomotic leakage in $3 / 35$ patients $(8.6 \%$; excluding 3 patients with Miles' operation and 12 patients who had construction of a covering stoma during surgery), anastomotic stricture in $3 / 47$ patients $(6.4 \%$; excluding 3 patients with Miles' operation), postoperative ileus in $3 / 50$ patients $(6.0 \%)$, and conversion to conventional open laparotomy in $1 / 50$ patients $(2.0 \%)$. Construction of a covering stoma was carried out during surgery in $12 / 47$ patients [25.5\%; 0/16 Rs patients $(0.0 \%), 3 / 20 \mathrm{Ra}$ patients $(15.0 \%)$ and $9 / 11 \mathrm{Rb}$ patients $(81.8 \%)]$. The mean postoperative hospital stay was 19.1 days, range 9-167 days (Table III).

\section{Discussion}

The internal approach that places priority on managing the vessels is the mainstream of pure LACS in Japan, but the lateral approach is often employed with hybrid 2-port HALS as with conventional open laparotomy to initially mobilize the colon/rectum from the paracolic gutter. This enables all the assistants, students, and attending staff to see the surgical field on the same monitor which occurs with pure LACS. With pure LACS, two-dimensional endoscopic procedures are mostly performed on the monitor, whereas the three-dimensional architecture of the intraperitoneal region can be performed more safely and surely with HALS than with pure LACS since the procedure is assisted by the left-hand of the operator as well as the monitor. There exists only 6 patterns with respect to the position of the patient, port sites, and operating room setting, and it would be relatively easy for a certified member of the Japanese Society of Digestive Surgery, who has performed hybrid 2-port HALS in at least 10 patients, to acquire the skills required for performing colon cancer surgery, including hemicolectomy, transverse colectomy, and sigmoidectomy. In the event of complications with splenocolic ligament detachment, splenic hemorrhage, or difficulty in achieving ligation/dissection of the middle colic vein, a hand access site is planned for colon cancer surgery at a site where emergency manipulation can be carried out in an open fashion through a small incision. In patients with progressive rectal cancer, it is possible to make a longitudinal incision of $40-55 \mathrm{~mm}$ in the lower abdomen from the beginning in anticipation of extending the incision. Hybrid 2-port HALS, therefore, may be considered useful for shortening the learning curve, at least during the introduction of LACS for patients with colon cancer.

In contrast, laparoscopy-assisted rectal cancer surgery is quite difficult compared with that for colon cancer, and the indications and methods employed markedly vary between hospitals. In the more than 50 patients that we have treated to date using laparoscopy-assisted rectal cancer, a 3-port procedure was employed for the initial few patients, but a 2port procedure is definitely feasible at present as for colon cancer surgery. A small transverse incision of $\sim 45-55 \mathrm{~mm}$ is made at the superior border of the pubic bone in advance to reach the deep pelvic floor via HALS, and non-pneumoperitoneum/laparoscopy-assisted manipulation and open surgery under direct vision are combined.

The most important procedure of rectal transection and anastomosis is performed while viewing the monitor. i) In patients with Rs cancer, a large Satinski clamp (esophageal clamp) is placed across the rectum horizontal to the pubic bone in the direction of the minor rectal axis. After sufficient lavage of the rectum, a curved cutter (Green-cartridge) is used for rectal transection directly parallel to the clamp, followed by ECS33 with DST and complete closure of the mesocolon, the mesorectum, and the pelvic floor peritoneum. ii) In patients with Ra cancer, a large Satinski clamp is used to clamp the rectum in the direction of Rs, and transection with a curved cutter is carried out after intrarectal irrigation. Prior to DST, all layers of the stump are additionally sutured with 2-3 reinforcing stitches ( $\leq 6$ stitches in total) at both corners, using absorbable 3-0 Vicryl attached to a needle. An air leak test is performed after the completion of ECS33 with 
DST, and then the mesocolon, mesorectum, and pelvic floor peritoneum are closed. iii) In patients with $\mathrm{Rb}$ cancer, a large Satinski clamp is used to clamp the rectum in the direction of its minor axis and orthogonal to the pubic bone (anterior to posterior), unlike clamping for Rs or Ra cancer. After intrarectal irrigation, not more than two transections are carried out along the clamp using an Endopath stapler (Ethlon 60-mm Green/Gold cartridge), while grasping and extending the clamped rectum in the cranial direction so that the transected pararectal tissue does not become sharply cone-shaped. Following ECS33 with DST (IO anastomosis), the mesocolon, mesorectum and pelvic floor peritoneum are completely closed.

In patients with cancer arising from the lower rectum, a covering stoma such as a temporary loop ileostomy or colostomy is often constructed even after routine open laparotomy to avoid fatal complications such as leakage from a low anastomosis (18-21). When LACS is performed, a covering colostomy is frequently constructed for the following patients: i) patients in whom DST was performed after 2 or more mechanical transections of the lower rectum, ii) patients with excessive tension at the anastomotic site after it has been pulled through the pelvic floor, and iii) patients with a poor blood supply to the anastomotic site (15). When low rectal anastomosis is conducted below the peritoneal reflection, pelvic floor peritonitis due to suture disruption is a serious potential complication that has been encountered by all colorectal surgeons $(22,23)$. However, aside from the issue of when and where to place the stoma, it is well known that constructing a loop colostomy rapidly improves a septic patient. It has, therefore, been argued by some researchers that a prophylactic diverting loop colostomy should be actively constructed after lower rectal anastomosis (15,19-21). Leakage of a low anastomosis is presumed to occur because of minor separation at the suture line due to mechanical traction-induced anastomoses in the perineal region by sitting up during the early postoperative period and anastomotic rupture due to a sudden increase in intrarectal pressure associated with accumulation of a large volume of intrarectal gas. Reinforcement of the anastomosis is considered to be the most effective countermeasure for physical/mechanical factors (15). To safely avoid construction of a covering stoma in patients with advanced lower rectal cancer, trans-anal reinforcing sutures (TARS) and posterior partial anal sphincterotomy (PPAS) to prevent an increase in pressure inside the rectum/anal canal have been added after completion of a low anastomosis by routine DST reconstruction in patients treated by LACS, and good results have been achieved (15). Quality of life is undoubtedly improved when patients do not have to manage a stoma. Some patients require a covering loop colostomy even after standard laparotomy depending on various factors, so it cannot be argued that a covering colostomy should never be constructed when less invasive LACS is performed. In the actual clinical setting, it is very complicated to construct a low anastomosis in elderly patients with concomitant diseases and male patients with a narrow pelvis and high body mass index (BMI). A covering colostomy is therefore constructed in a relatively large number of situations. Also, in patients with advanced low rectal cancer, metastasis/recurrence may occur at the anastomotic site and in the pelvic cavity such as on the anterior surface of the sacrum at a relatively early period after construction of a covering colostomy. Chemoradiotherapy can be administered effectively in some of these patients due to the presence of a loop colostomy. Patients with a poor prognosis who develop distal metastasis/recurrence in the liver or lungs, and elderly patients with concomitant diseases are usually hospitalized again after 3-6 months to close the stoma, except for patients in whom closure will lead to surgical stress and is not advantageous $(23,24)$. In consideration of the basic concept of LACS, it is more apparent than with typical laparotomy that not constructing a stoma is best, and efforts should be made to avoid this when possible.

Chemotherapy is often combined with radiation therapy to treat patients in Western counries with progressive rectal cancer, but it has been extensively argued in Japan that lateral lymph node metastasis should be managed by lateral lymph node dissection (25-27). Although many reports have indicated that a better prognosis is obtained by lateral lymph node dissection in patients with lateral lymph node metastasis, opinions vary concerning prophylactic bilateral lymph node dissection, and a consensus is yet to be established (28-30). Making a transverse mini-incision of $55 \mathrm{~mm}$ at the superior border of the pubic bone has been the standard procedure for patients with advanced low rectal cancer, but open hemilateral lateral lymph node dissection of metastatic lymph nodes would be a satisfactory procedure that could be achieved by making a small incision near the lymph nodes for dissection as a hand access site, such as a midline incision or pararectal incision in the lower abdomen.

In this investigation of colon cancer surgery, no patient required conversion to conventional laparotomy or developed anastomotic leakage, and good results were obtained with a relatively short operating time, short postoperative hospital stay, and a small amount of bleeding. Postoperative complications included wound infection in $5 / 58$ patients $(8.6 \%$, initially $3 / 20$ patients) and postoperative ileus in $3 / 58$ patients $(5.2 \%)$.

In contrast, the operating time and postoperative hospital stay were longer and the blood loss was greater with rectal cancer surgery than with colon cancer surgery. Super LAR/LAR for low rectal cancer is considered particularly difficult among hybrid 2-port HALS, but the blood loss was small and the hospital stay was shorter in all 3 patients who underwent Miles' operation. Complications included wound infection in $6 / 50$ patients $(12.0 \%$, initially $4 / 20$ patients), anastomotic leakage in $3 / 35$ patients $(8.6 \%)$, anastomotic stricture in $3 / 47$ patients $(6.4 \%)$, postoperative ileus in $3 / 50$ patients $(6.0 \%)$, and conversion to open laparotomy in $1 / 50$ patients $(2.0 \%)$. However, a second operation due to secondary hemorrhage, late small intestinal perforation, or ureteric injury was not required in any of our patients who underwent colorectal cancer surgery. More patients should be investigated in the future, including patients with advanced low rectal cancer, to enable assessment of the stoma construction rate during surgery for $\mathrm{Rb}$ rectal cancer and detailed comparison with standard laparotomy to determine the long-term outcome, including bowel and anal function and the local recurrence rate at intrapelvic sites such as the anastomosis and the anterior surface of the sacrum. 


\section{Acknowledgements}

This study was supported by grants from the Occult Neoplastic Cells Research and Study Group (\#2009-5007; Tokai University Hachioji Hospital, Hachioji, Tokyo, Japan) and the Research and Study Program of Tokai University Educational System General Research Organization (\#2007-04; Tokai University Hospital, Isehara, Kanagawa, Japan).

\section{References}

1. Mukai M, Tokunaga N, Ishida H, Makuuchi H, Tajima T and Mitomi T: Clinical experiences with laparoscopic colectomy. Dig Endosc 9: 11-15, 1997.

2. Mukai M, Okamoto Y, Oida Y, Mukoyama S, Ito I, Nakasaki H, Kawai K, Sato S and Makuuchi H: Endoscopic mucosal resection for superficially spreading colonic neoplasms larger than $5 \mathrm{~cm}$ in the right colon after dilute sodium hyaluronate injection: Report of two cases. Endoscopy 35: 973-975, 2003.

3. Makuuchi M and Sugihara K: Knacks \& Pit Falls: Surgery of the Colon, Rectum and Anus. 2nd edition, Bunkoudou Co., Ltd., Tokyo, 2004

4. Guideline of Large Bowel Cancer in Japan. Japanese Society for Cancer of the Colon and Rectum, Tokyo, 2005.

5. Mukai M, Tanaka A, Tajima T, Yamagiwa T, Okada K, Fukasawa M, Sato K, Oida Y, Nakamura M and Makuuchi H: Two-port hand-assisted laparoscopic surgery in 2-stage treatment for complete obstruction by left colon cancer: A case report. Oncol Rep 19: 875-879, 2008.

6. Romanelli JR, Kelly JJ and Litwin DE: Hand-assisted laparoscopic surgery in United States: an overview. Semin Laparosc Surg 8: 96-103, 2001

7. Nakajima K, Lee SW, Cocilovo C, Foglia C, Kim K, Sonoda T and Milson JW: Hand-assisted laparoscopic colorectal surgery using GelPort. Surg Endosc 18: 102-105, 2004

8. Nakajima K, Lee SW, Cocilovo C, Foglia C, Sonoda T and Milson JW: Laparoscopic total colectomy: hand-assisted vs. standard technique. Surg Endosc 18: 582-586, 2004.

9. Yamaguchi Y, Minami K, Kawabuchi Y, Emi M and Toge T: Anterior resection of rectal cancer through a one hand-size incision with or without laparoscopy: Proposal of one hand-size incision surgery (OHaSIS). J Surg Res 129: 136-141, 2005.

10. Jacobs M, Verdja JC and Goldstein HS: Minimally invasive colon resection (laparoscopic colectomy). Surg Laparosc Endosc 1: 144-150, 1991.

11. The Clinical Outcomes of Surgical Therapy Study Group: A comparison of laparoscopically assisted and open colectomy for colon cancer. N Engl J Med 350: 2050-2059, 2004.

12. Fukunaga M, Kidokoro A, Iba T, Sugiyama K, Fukunaga T, Nagakari K, Suda M and Yoshikawa S: Laparoscopy assisted low anterior resection with a prolapsing technique for low rectal cancer. Surg Today 35: 598-602, 2005

13. 9th Nationwide Survey of Endoscopic Surgery. J Japan Soc Endoscopic Surg 13: 529-539, 2008

14. Statistical Analyses of Organization of the Ministry of Health, Labour and Welfare Ministry; data base system, 2007. http:// wwwdbtk.mhlw.go.jp/IPPAN/ippan/scm_K_Ichiran.

15. Mukai M, Fukasawa M, Kishima K, IIzuka S, Fukumitsu H, Yazawa N, Tajima T, Nakamura M and Makuuchi H: Transanal reinforcing sutures after double stapling for low rectal cancer: Report of two cases. Oncol Rep 21: 335-339, 2009.
16. Mukai M, Kishima K, Iizuka S, Fukumitsu H, Fukasawa M, Yazawa N, Tajima T, Nakamura M and Makuuchi H: Two-port hand-assisted laparoscopic surgery for 2-stage treatment of complete obstruction by distal sigmoid colon cancer: A case report. Oncol Rep 21: 1203-1208, 2009.

17. Mukai M, Kishima K, Iizuka S, Fukumitsu H, Fukasawa M, Yazawa N, Tajima T, Nakamura M and Makuuchi H: Curative resection by hybrid 2-port HALS in a patient with advanced cecal cancer invading the urinary bladder: A case report. Oncol Rep 21: $1385-1389,2009$

18. Pakkastie TE, Ovaska JT, Pekkara ES, Luukkonen PE and Jarvinen HJ: A randomized study of colostomies in low rectal anastomoses. Eur J Surg 163: 929-933, 1997.

19. Dehni N, Schlegel RD, Cunningham C, Guiguet M, Tiret E and Pare R: Influence of a defunctioning stoma on leakage rates after low rectal anastomosis and colonic $\mathrm{J}$ pouch-anal anastomosis. Br J Surg 85: 1114-1117, 1998.

20. Tschmelitsch J, Wykypiel H, Prommegger R and Bodner E: Colostomy vs tube cecostomy for protection of low anastomosis in rectal cancer. Arch Surg 134: 1385-1388, 1999.

21. Rullier E, Le Toux N, Laurent C, Garrelon J-L, Parneix M and Saric J: Loop ileostomy versus loop colostomy for defunctioning low anastomoses during rectal cancer surgery. World J Surg 25: 274-278, 2001.

22. Karanjia ND, Corder AP, Holdsworth PJ and Heald RJ: Risk of peritonitis and fatal septicaemia and the need to defunction the low anastomosis. Br J Surg 78: 196-198, 1991.

23. Gooszen AW, Geelkerken RH, Hermans J, Lagaay MB and Gooszen HG: Temporary decompression after colorectal surgery: randomized comparison of loop ileostomy and loop colostomy. Br J Surg 85: 76-79, 1998.

24. Mukai M, Himeno S, Mukoyama S, Tajima T, Saito Y, Ito I, Nakasaki H, Sato S and Makuuchi H: Is the temporally loop colostomy in the right transverse colon appropriate for complete obstruction by colorectal cancer? Oncol Rep 10: 693698, 2003.

25. NIH consensus conference: Adjuvant therapy for patients with colon and rectal cancer. JAMA 264: 1444-1450, 1990.

26. Krook JE, Moertel CG, Gunderson LL, Wieand HS, Collins RT, Beart RW, Kubista TP, Poon MA, Meyers WC, Mailliard JA, Twito DI, Morton RF, Veeder MH, Witzig TE, Cha S and Vidyarthi SC: Effective surgical adjuvant therapy for high-risk rectal carcinoma. N Engl J Med 324: 709-715, 1991.

27. Wolmark N, Wieand HS, Hyams DM, Colangelo L, Dimitrov NV, Romond EH, Wexler M, Prager D, Cruz AB Jr, Gordon PH, Petrelli NJ, Deutsch M, Mamounas E, Wickerham DL, Fisher ER, Rockette H and Fisher B: Randomized trial of postoperative adjuvant chemotherapy with or without radiotherapy for carcinoma of the rectum: National Surgical Adjuvant Breast and Bowel Project Protocol R-02. J Natl Cancer Inst 92: 388-396, 2000.

28. Moreira LF, Hizuta A, Iwagaki H, Tanaka N and Orita K: Lateral lymph node dissection for rectal carcinoma below the peritoneal reflection. Br J Surg 81: 293-296, 1994.

29. Hida J, Yasutomi M, Fujimoto K, Maruyama T, Okuno K and Shindo K: Dose lateral lymph node dissection improve survival in rectal carcinoma? Examination of node metastases by the clearing method. J Am Coll Surg 184: 475-480, 1997.

30. Sato H, Maeda K, Maruta M, Matsumori K and Koide Y: Who can get the beneficial effect from lateral lymph node dissection for Dukes C rectal carcinoma below the peritoneal reflection? Dis Colon Rectum 49: 3-12, 2006. 